\title{
ADAPTACIÓN DE LOS ESTUDIOS DE MAGISTERIO AL EEES: LAS TIC EN LOS NUEVOS PLANES DE ESTUDIO
}

\author{
ADAPTATION OF TEACHER TRAINING DEGREES TO THE EHEA: \\ ICT IN THE NEW STUDY PLANS
}

\author{
Rosario Isabel Herrada Valverde; rherrada@um.es \\ Facultad de Educación, Universidad de Murcia \\ Gabriel Herrada Valverde; u101421@usal.es \\ Facultad de Educación, Universidad de Salamanca
}

\section{RESUMEN}

Este artículo analiza el proceso de adaptación de los antiguos títulos de Maestro a los nuevos Grados adaptados al Espacio Europeo de Educación Superior en las universidades públicas españolas y, en particular, la presencia de asignaturas relacionadas con las Tecnologías de la Información y la Comunicación. El análisis de los datos recopilados muestra como este proceso ha dado lugar a una dispersión de la presencia de estas asignaturas dependiendo de cada universidad y especialidad.

PALABRAS CLAVE: Espacio Europeo de Educación Superior, Estudios de Magisterio, Planes de Estudio, Tecnologías de la Información y la Comunicación.

\section{ABSTRACT}

This paper analyzes the adaptation process of the old preservice teacher degrees to the new ones adapted to the European Higher Education Area, in particular, about the presence of subjects related with Information and Communication Technologies. The analysis of the collected data shows that this process has involved dispersion in the presence of these subjects according to the university and speciality.

KEYWORDS: European Higher Education Area, Study Plans, Teacher Training Degrees, Information and Communication Technologies. 


\section{INTRODUCCIÓN}

En Junio de 1999, los Ministros con competencias en Educación Superior de 29 países, incluida España, suscribieron la Declaración de Bolonia, que sentaba las bases del Espacio Europeo de Educación Superior (EEES), cuyo objetivo final era facilitar la movilidad de los ciudadanos, las posibilidades de obtener empleo y, en suma, fomentar el desarrollo del continente europeo. Actualmente, 47 países forman parte del EEES.

Una de las consecuencias directas de la implantación del EEES ha sido el diseño de nuevos planes de estudio de grado y máster, que sustituyen a las diplomaturas, licenciaturas e ingenierías. En el caso español, dicha adaptación se ha llevado a cabo de forma conjunta entre las diferentes universidades y la Agencia Nacional de Evaluación de la Calidad y Acreditación (ANECA).

En referencia a los estudios conducentes a la profesión de maestro, cabe indicar que de las siete especialidades de magisterio existentes previamente (educación infantil, educación primaria, educación física, educación musical, educación especial, lengua extranjera, audición y lenguaje) sólo las dos primeras han sido adaptadas como grado.

Aunque en un principio la Declaración de Bolonia y sus documentos asociados no hacían mención explícita a las Tecnologías de la Información y la Comunicación (TIC), con posterioridad se han ido incorporando iniciativas en el sentido de incorporarlas en la enseñanza superior, con programas como elearning (2004), que promovía la integración efectiva de las TIC en los sistemas de educación y formación europeos. La presencia de las nuevas tecnologías en el entorno actual lleva a pensar que el peso cualitativo y cuantitativo de las asignaturas relacionadas con las TIC en los nuevos planes de estudio de ambas especialidades sea importante, ya que sus alumnos serán los maestros de las futuras generaciones. Una vez iniciado el curso académico 2010/2011, momento en el cual todas las universidades públicas han implantado los nuevos estudios de grado, presentamos una visión general sobre el proceso de adaptación de los Títulos Oficiales que habilitan para ejercer la profesión de maestro, y en particular sobre la inclusión de asignaturas relacionadas con las TIC en los nuevos Grados de Educación Infantil y Educación Primaria.

\section{FUNDAMENTACIÓN TEÓRICA}

\subsection{Proceso de adaptación al EEES de las nuevas titulaciones oficiales}

El proceso de adaptación al EEES ha supuesto, entre otras, las siguientes novedades:

- La adopción de un sistema de tres ciclos de estudios, que conducen al Título de Graduado, de Máster Universitario y de Doctor (Real Decreto 1393/2007, de 29 de octubre). Los créditos asignados en los ciclos de estudios para la adquisición del título de Graduado es de 240 créditos, mientras que para el Máster oscila entre 60 y 120 créditos.

- La adopción del sistema europeo de créditos ECTS (European Credit Transfer and Accumulation System) como unidad de medida del trabajo del estudiante (Real Decreto 1125/2003, de 5 de septiembre), en la que un crédito ECTS corresponde a 25-30 horas de trabajo del estudiante. Esto implica un cambio 
metodológico ya que se pasa de una enseñanza centrada en la adquisición de conocimientos a una enseñanza enfocada al aprendizaje de los estudiantes.

- La emisión del Suplemento Europeo al Título (Real Decreto 1044/2003, de 1 de agosto), que permite a los titulados universitarios que sus títulos vayan acompañados de un documento de información sobre el nivel y contenido de las enseñanzas que hayan cursado en su universidad, con las ventajas que ello comporta para su movilidad académica y profesional en otras universidades y países europeos.

- Consideración de informes internacionales. Así, por ejemplo, entre los años 2003 y 2006, se promovieron en España experiencias piloto de diseño e implantación de planes de estudio y títulos de grado a través del trabajo en red de las universidades españolas con el objetivo de planificar un marco nacional de titulaciones compatible con el futuro "marco europeo" (Marco de Cualificaciones, 2005) siguiendo las recomendaciones y el sistema de trabajo utilizadas en el proyecto Tuning (2003). La memoria final de estas experiencias se publicó como "Libro Blanco" (ANECA-Libros-Blancos, 2010).

- La evaluación y acreditación por parte de la ANECA de los nuevos títulos oficiales adaptados al EEES en las diferentes universidades españolas. Dicho proceso ha consistido en la verificación previa por parte de los organismos competentes según unos protocolos específicos, y la posterior evaluación del título por parte de la ANECA a través del programa Verifica (ANECA-VERIFICA, 2010). Tras la evaluación de dichos títulos, la ANECA remite un informe al Consejo de Universidades, el cual, en caso de que el informe recibido sea favorable, dictará la resolución de verificación, que se comunica al Ministerio de Educación, a la Comunidad Autónoma correspondiente y a la Universidad. Tras la autorización de la Comunidad Autónoma, el Ministerio de Educación eleva al Gobierno la propuesta para el establecimiento del carácter oficial del título y su inscripción en el Registro de Universidades, Centros y Títulos, RUCT (Real Decreto 1509/2008, de 12 de septiembre), cuya aprobación mediante Acuerdo del Consejo de Ministros se publica en el Boletín Oficial del Estado. El registro en el RUCT implica que el Título ha adquirido la acreditación, y por tanto, puede ser puesto en funcionamiento por un período de 6 años desde su fecha de registro en el RUCT, tras lo cual tendrá que volver a ser verificado para mantener la acreditación.

\subsection{Configuración de los estudios que habilitan para el ejercicio de la profesión de maestro}

En España, los estudios universitarios adaptados al EEES que permiten ejercer la profesión de maestro son dos Grados, Educación Infantil y Educación Primaria. A efectos prácticos, esto ha supuesto la desaparición de las titulaciones de Maestro en Educación Física, Educación Musical, Lengua Extranjera, Educación Especial, Audición y Lenguaje, si bien se contempla la posibilidad de incorporar competencias docentes especificas relacionadas con dichas especialidades en los nuevos grados (ANECA-Libros-Blancos, 2010). Para algunos, la decisión de adaptar únicamente estas dos especialidades en detrimento de la formación de los especialistas significa una oportunidad perdida para afrontar la realidad de las escuelas y favorecer una formación inicial del profesorado que atiende a las exigencias de la sociedad 
del siglo XXI (Reyes-López, 2010). Esto ha provocado, a su vez, numerosas dudas e inquietudes en los estudiantes que están cursando los estudios de magisterio en las especialidades a extinguir (Alonso-Martín, 2008). El motivo por el cual la mayoría de las universidades se mostraron favorables a la implantación de un único título de Grado de Educación Infantil y uno de Educación Primaria se debió, en parte, a las conclusiones de la Red de Magisterio creada para la elaboración del Libro Blanco de las Titulaciones de Magisterio, que determinó que, dentro del mapa europeo, el sistema anterior de siete especialidades era exclusivo del sistema español, y que había un gran desequilibrio en la oferta y demanda de las mismas (Maldonado-Rico, 2004).

Por otro lado, cabe señalar que mientras que la denominación de "Maestro en Educación Infantil" y "Maestro en Educación Primaria" era homogénea para todo el territorio nacional, el Real Decreto 1393/2007, de 29 de Octubre, ofrece autonomía a las universidades para establecer las denominaciones de los nuevos grados. Así, aunque en este documento hablaremos de Grados en Educación Infantil/Primaria, las nuevas denominaciones incluyen: Graduado en Educación Infantil/Primaria, Graduado en Maestro de Educación Infantil/Primaria, Graduado en Maestro en Educación Infantil/Primaria, Graduado en Magisterio de Educación Infantil/Primaria, Graduado en Magisterio en Educación Infantil/Primaria.

Por el contrario, la duración de los títulos se ha homogeneizado, ya que mientras los antiguos títulos de Maestro oscilaban entre 180 y 219 créditos LRU según cada universidad, los nuevos Grados tienen una duración de 240 créditos ECTS (Resolución de 17 de diciembre de 2007). Además, existe legislación específica (Orden ECI/3854/2007, de 27 de diciembre) que obliga a que el plan de estudios de los Grados de Educación Infantil incluyan, como mínimo, 100 créditos de formación básica, 60 créditos de formación didáctica y disciplinar, y 50 créditos de prácticum. De igual forma, el plan de estudios de los Grados de Educación Primaria deberán incluir, como mínimo, 60 créditos de formación básica, 100 créditos de formación didáctica y disciplinar, y 50 créditos de prácticum (Orden ECI/3857/2007, de 27 de diciembre). Asimismo, se establece la posibilidad de proponer menciones cualificadoras de entre 30 y 60 ECTS, adecuadas a los objetivos, ciclos y áreas de la Educación Infantil y de la Educación Primaria, teniendo en cuenta lo establecido al respecto en la Ley Orgánica 2/2006, de 3 de mayo, de Educación (LOE).

\subsection{Normativa sobre las TIC en las etapas de Educación Infantil y Primaria}

Las TIC están transformando el aula y las funciones docentes, y estos cambios están induciendo cambios sistemáticos en las teorías y prácticas didácticas (Fernández-Muñoz, 2003). Diferentes autores han destacado la necesidad de adaptar los procesos de enseñanzaaprendizaje al EEES mediante el desarrollo de nuevos planes de estudio y a través del uso de nuevas metodologías docentes, entre las que las TIC juegan un papel destacado como recurso didáctico, elemento para la comunicación y la expresión, e instrumento para la organización, gestión y administración educativa (Ferro-Soto y col., 2009). En esta línea, algunos autores han alertado sobre la necesidad de apostar más claramente por la implementación de las TIC en la labor docente de los profesores (Castañeda, 2009).

En su preámbulo, la LOE señala que la Unión Europea y la UNESCO han propuesto una serie de líneas de actuación parar mejorar la calidad y la eficacia de los sistemas de educación y de formación, entre las que se incluye el hecho de garantizar el acceso de todos a las TIC, además de hacer mención específica, en su Artículo 157.f, a la formación del profesorado en 
las TIC. Asimismo, el proyecto Tuning (2003) incluye las nuevas tecnologías de la información como habilidades y destrezas transversales. Resulta evidente que, para poder llevar a buen puerto las normativas previas, los futuros maestros deben ser formados en las TIC en su formación universitaria.

En referencia a la Educación Infantil, el Artículo 14.5 de la LOE señala que las Administraciones Educativas fomentarán una primera aproximación, entre otras, a las TIC. EI Real Decreto 1630/2006, de 29 de diciembre, por el que se establecen las enseñanzas mínimas del segundo ciclo de Educación Infantil incluye a las TIC como una forma de comunicación y representación, junto con el lenguaje verbal, el lenguaje artístico, el lenguaje corporal y el lenguaje audiovisual, e incluye un bloque de contenidos específico denominado "Lenguaje audiovisual y tecnologías de la información y la comunicación".

En cuanto a la Educación Primaria, el Artículo 17.i de la LOE y Artículo 3.i del Real Decreto 1513/2006, de 7 de diciembre, por el que se establecen las enseñanzas mínimas de la Educación Primaria, incluyen como objetivo de la Educación Primaria la iniciación, para el aprendizaje, de las TIC desarrollando un espíritu crítico ante los mensajes que reciben y elaboran, mientras que el Artículo 19.2 de la LOE y el Artículo 4.5 del Real Decreto 1513/2006 se indica que las TIC se trabajarán de forma transversal en todas las áreas. Además, el Real Decreto 1513/2006, en su anexo I, incluye como competencia básica el "Tratamiento de la información y competencia digital", que implican ser una persona autónoma, eficaz, responsable, crítica y reflexiva al seleccionar y utilizar la información y sus fuentes, así como las distintas herramientas tecnológicas, así como tener una actitud crítica y reflexiva en la valoración de la información disponible. Estas y otras competencias son tratadas en profundidad en el estudio de Prendes-Espinosa (2010).

\section{METODOLOGÍA DEL ESTUDIO}

La metodología de esta investigación ha consistido en la búsqueda, recopilación y análisis de información referente al proceso de implantación de nuevos Grados de Educación Infantil y Educación Primaria, y su comparación con los antiguos Magisterios de ambas especialidades. Las fuentes de información utilizadas han sido:

i. Documentación disponible sobre la declaración de Bolonia en el marco internacional (comunicados, declaraciones, convenios, etc.); legislación promulgada en España para la adaptación al EEES; documentos de difusión de información sobre el proceso de adaptación al EEES.

ii. Documentación de la ANECA (libros blancos, programa Verifica, etc.).

iii. Documentación de páginas web de las universidades públicas sobre los antiguos planes de estudio de magisterio así como de los nuevos grados.

El proceso de recopilación, organización y análisis de la información se ha dividido en las siguientes fases:

I. Recopilación y organización de la información:

A. Referente a la creación y desarrollo del Proceso de Bolonia, reuniones posteriores, así como documentos legislativos y divulgativos a nivel nacional. Dicha información ha 
sido agrupada dentro del bloque temático "Documentación legislativa sobre el EEES", haciendo uso del procesador de textos Word.

B. Referente a la legislación española sobre el proceso de adaptación al EEES. Información sobre los libros blancos de los Grados de Educación infantil y Educación Primaria según la ANECA, así como del programa Verifica desarrollado por dicho organismo. Esta información se ha agrupado en el bloque temático "Documentación ANECA", utilizando Word.

C. Referente a los planes de estudio de Educación Infantil y Educación Primaria, a partir de las páginas web de las universidades públicas españolas y en su caso en Boletín Oficial del Estado (B.O.E.). Esta información se ha agrupado dentro del bloque temático "Asignaturas TIC en Educación Infantil y Educación Primaria", haciendo uso de Word y Excel. Dicho bloque temático se ha dividido a su vez en dos bloques: "TIC en los antiguos magisterios", con información sobre las titulaciones de Maestro en Educación Infantil y Maestro en Educación Primaria; y "TIC en los nuevos grados" con información referente al Grado en Educación Infantil (o denominación análoga) y al Grado en Educación Primaria (o denominación análoga). Aunque cualquier asignatura puede tratar el uso de las TIC de forma transversal, ha sido necesario establecer un conjunto de palabras clave cuya presencia en el nombre de las asignaturas determinarán si se encuadran o no dentro del ámbito de las TIC. En concreto, las siguientes palabras clave utilizadas han sido: "Alfabetización digital", "Audiovisual", "Computacional", "Entornos digitales", "Imagen digital”, "Informática”, "Interactivo", "Internet", "Lenguaje informático", "Multimedia", "Nuevas tecnologías", "Ordenador", "Recursos tecnológicos", "Sociedad de la información", "Sociedad del conocimiento", "Tecnologías para el aprendizaje y el conocimiento (TAC)", "Tecnologías de la información", "TIC", "Televisión", "Video digital", "Videojuegos", "Virtual", "Virtualidad". En aquellas universidades que ofertan estos estudios en diferentes campus, se ha tomado como referencia el plan de estudios de uno de ellos.

II. Análisis de la información: Tras recopilar y organizar la información, se han analizado las medidas burocráticas promovidas para la implantación de los nuevos planes de estudio haciendo uso de la información agrupada en los núcleos temáticos "Documentación legislativa sobre el EEES" y "Documentación ANECA". Posteriormente, se ha analizado la presencia de asignaturas relacionadas con las TIC en los nuevos grados y se ha comparado con las antiguas titulaciones de magisterio haciendo uso de la información almacenada en el bloque temático "Asignaturas TIC en Educación Infantil y Educación Primaria", y sus respectivos sub-bloques. 


\section{LAS TIC EN LOS NUEVOS GRADOS DE EDUCACIÓN INFANTIL Y PRIMARIA. COMPARACIÓN CON LOS ANTÍGUOS TÍTULOS DE MAESTRO}

Para empezar, debemos comentar que tras la implantación del EEES, el nuevo mapa de titulaciones muestra como ha aumentado el número de universidades que ofertan dichos estudios. Así, mientras las titulaciones de Maestro en Educación Infantil y de Maestro en Educación Primaria eran ofertados por 38 y 37 universidades, respectivamente, ahora se ofertan un total de 39 Grados de Educación Infantil y Educación Primaria, ya que todas las universidades que ofrecían estos estudios de Maestro han llevado a cabo la adaptación al grado, y además la Universidad de La Rioja ha incorporado el Grado de Educación Primaria y la Universidad Rey Juan Carlos ha incorporado ambos grados, ya que antes no ofertaba estudios de Maestro. Esto demuestra la gran importancia y demanda de dichos estudios, en concordancia con investigaciones previas en las que se concluye que los estudiantes suelen cursar estudios de Magisterio por motivos vocacionales (Sánchez-Lissen, 2002) y por la elevada tasa de egresados que obtienen un trabajo (Maldonado-Rico, 2004). Esto contrasta con otras titulaciones que no han sido adaptadas al grado, como es el caso de la Licenciatura en Psicopedagogía, pese a que ha venido siendo la titulación de segundo ciclo más cursada por estudiantes que han finalizado su estudios de Magisterio (Maldonado-Rico, 2004).

En cuanto al análisis propiamente dicho de las asignaturas de TIC, la Tabla 1 resume toda la información recopilada sobre la presencia de dichas asignaturas en las diferentes universidades y especialidades, tanto de los nuevos grados como en los antiguos títulos de magisterio. Indicar que se ha marcado con un asterisco a aquellas universidades que, al concentrar la optatividad en los últimos cursos de grado, aun no han publicado su oferta. Así, por ejemplo, el plan de estudios de Educación Infantil de la Universidad Pública de Navarra incluye la mención "Documentación, comunicación audiovisual y digital y proyectos escolares" de 21 créditos ECTS, pero aun no han sido publicadas las asignaturas que componen dicha mención. 


\begin{tabular}{|c|c|c|c|c|c|c|c|c|c|}
\hline \multirow{3}{*}{ CCAA } & \multirow{3}{*}{$\begin{array}{l}\text { Universidad/ } \\
\text { Campus }\end{array}$} & \multicolumn{4}{|c|}{ MAESTRO } & \multicolumn{4}{|c|}{ GRADO } \\
\hline & & \multicolumn{2}{|c|}{ Infantil } & \multicolumn{2}{|c|}{ Primaria } & \multicolumn{2}{|c|}{ Infantil } & \multicolumn{2}{|c|}{ Primaria } \\
\hline & & $\begin{array}{c}\text { Troncal/ } \\
\text { Obligat }\end{array}$ & Optat. & $\begin{array}{c}\text { Troncal/ } \\
\text { Obligat }\end{array}$ & Optat. & Básica & Optat. & Básica & Optat. \\
\hline \multirow{8}{*}{ ANDALUC } & Almería & 1 & 3 & 1 & 3 & 0 & 3 & 0 & 3 \\
\hline & Cádiz & 1 & 2 & 1 & 2 & 0 & 0 & 0 & 1 \\
\hline & Córdoba & 1 & 0 & 1 & 0 & 1 & 1 & 2 & 0 \\
\hline & Granada & 1 & 0 & 1 & 0 & 1 & 0 & 1 & 0 \\
\hline & Huelva & 1 & 0 & 1 & 0 & 1 & 0 & 1 & 0 \\
\hline & Jaén & 1 & 0 & 1 & 0 & 0 & $5(\mathrm{M})$ & 0 & 0 \\
\hline & Malaga & 1 & 0 & 1 & 1 & 0 & 0 & 1 & 0 \\
\hline & Sevilla & 1 & 0 & 1 & 1 & 1 & 0 & 1 & 0 \\
\hline ARAGÓN & Zaragoza & 1 & 0 & 1 & 0 & 0 & $0 *$ & 1 & $0 *$ \\
\hline ASTURIAS & Oviedo & 1 & 1 & 1 & 1 & 1 & $3(\mathrm{M})$ & 1 & $3(\mathrm{M})$ \\
\hline BALEARES & Mallorca & 1 & 1 & 1 & 1 & 0 & 1 & 1 & $4(\mathrm{M})$ \\
\hline \multirow{2}{*}{ CANARIAS } & La Laguna & 1 & 1 & 1 & 1 & 1 & 1 & 0 & 2 \\
\hline & Las Palmas & 1 & 3 & 1 & 3 & 1 & 1 & 1 & 2 \\
\hline CANTABR & Santander & 1 & 1 & 1 & 1 & 1 & 3 & 1 & 4 \\
\hline \multirow{4}{*}{ CAS-LEÓN } & Burgos & 1 & 2 & 1 & 2 & 1 & 1 & 1 & 0 \\
\hline & León & 1 & 0 & 1 & 0 & 1 & 0 & 1 & 0 \\
\hline & Salamanca & 1 & 0 & 1 & 2 & 1 & 3 & 1 & 3 \\
\hline & Valladolid & 1 & 2 & 1 & 1 & 1 & 0 & 1 & 1 \\
\hline CAS-LM & Toledo & 1 & 0 & 1 & 0 & 0 & 0 & 0 & 0 \\
\hline \multirow{5}{*}{ CATALUÑA } & Autónoma B. & 1 & 1 & 1 & 1 & 0 & 2 & 0 & 3 \\
\hline & Barcelona & 1 & 2 & 1 & 2 & 1 & 0* & 0 & 0* \\
\hline & Girona & 1 & 2 & 1 & 2 & 0 & $11(\mathrm{M})$ & 0 & $11(\mathrm{M})$ \\
\hline & Lleida & 1 & 6 & 1 & 6 & 0 & $0 *$ & 0 & $0^{*}$ \\
\hline & Rovira i Virgili & 1 & 0 & 1 & 0 & 0 & $0 *$ & 0 & $0 *$ \\
\hline EXTREM & Badajoz & 1 & 2 & 1 & 2 & 1 & 0 & 1 & 0 \\
\hline \multirow{3}{*}{ GALICIA } & Coruña & 1 & 0 & 1 & 1 & 1 & 2 & 1 & 0 \\
\hline & Santiago & 1 & 0 & 1 & 0 & 1 & 0 & 1 & 3 \\
\hline & Vigo & 1 & 1 & 1 & 1 & 1 & 0 & 1 & 2 \\
\hline LA RIOJA & Logroño & 1 & 0 & --- & --- & 0 & $0^{*}$ & 0 & $0^{*}$ \\
\hline \multirow{4}{*}{ MADRID } & Alcalá Henar. & 1 & 0 & 1 & 0 & 0 & $0^{*}$ & 0 & $0 *$ \\
\hline & Autónoma M. & 1 & 0 & 1 & 0 & 1 & $5(\mathrm{M})$ & 1 & $8(\mathrm{M})$ \\
\hline & Complutense & 1 & 1 & 1 & 1 & 0 & 1 & 0 & 2 \\
\hline & Rey J.C. & --- & --- & --- & --- & 2 & 0* & 2 & 0* \\
\hline MURCIA & Murcia & 1 & 0 & 1 & 1 & 1 & 0 & 1 & 3 \\
\hline NAVARRA & Pamplona & 1 & 1 & 1 & 1 & 1 & $0^{*}$ & 1 & $0^{*}$ \\
\hline PAIS-VASC & Bilbao & 1 & 1 & 1 & 1 & 1 & 1 & 1 & 2 \\
\hline \multirow{3}{*}{ VALENCIA } & Alicante & 1 & 0 & 1 & 0 & 0 & 0 & 1 & 0 \\
\hline & Castellón & 2 & 0 & 2 & 0 & 1 & 1 & 1 & 1 \\
\hline & Valencia & 1 & 2 & 1 & 2 & 0 & 0 & 0 & $6(\mathrm{M})$ \\
\hline \multicolumn{2}{|c|}{ TOTAL } & 39 & 35 & 38 & 40 & 24 & 45 & 27 & 64 \\
\hline
\end{tabular}

Tabla 1. Asignaturas TIC en los nuevos Grados de Magisterio. 
El primer elemento que llama claramente la atención es la heterogeneidad de la presencia de las TIC en los nuevos planes de estudio. Así, mientras que los antiguos títulos de Maestro contaban en ambas especialidades con la asignatura troncal denominada "Nuevas Tecnologías Aplicadas a la Educación", los nuevos grados no toman de forma común ninguna asignatura básica sobre dicha disciplina, quedando a criterio de cada universidad incluir materias relacionadas con las TIC. Así, se observa que hay 10 universidades públicas españolas en las cuales los nuevos Grados de Educación Infantil no incluyen asignaturas directamente relacionadas con las TIC (Cádiz, Málaga, Zaragoza, Castilla-La Mancha, Lleida, Rovira i Virgili, Logroño, Alcalá de Henares, Alicante y Valencia), hecho que también sucede en 7 Grados en Educación Primaria (Jaén, Castilla-La Mancha, Barcelona, Lleida, Rovira i Virgili, Logroño y Alcalá de Henares). Por el contrario, el resto de universidades si incluyen asignaturas relacionadas con las TIC, e incluso cuatro universidades ofrecen menciones específicas sobre TIC en el Grado de Educación Infantil (Jaén: "Tecnologías de la Comunicación y La Información"; Oviedo: "Tecnología y Comunicación Educativa"; Girona: "Tecnologies de la informació i la comunicació (3-12)"; Autónoma de Madrid: "Nuevos entornos de Aprendizaje") y cinco menciones en el Grado en Educación Primaria (Oviedo: "Tecnología y Comunicación Educativa"; Illes Balears: "Tecnología educativa"; Girona: "Tecnologies de la informació i la comunicació (3-12)"; Autónoma de Madrid: "Tecnologías de la información y la comunicación"; Valencia: "Tecnologías de la información y la comunicación").

Por otro lado, también se observa que no existe una correlación directa entre la presencia de asignaturas TIC en los antiguos magisterios y en los nuevos grados. Así, universidades como la Autónoma de Madrid, Cantabria, Illes Balears, Oviedo, etc. han aumentado notablemente su oferta de asignaturas TIC, en otras, como la de Cádiz o Lleida, se ha reducido considerablemente.

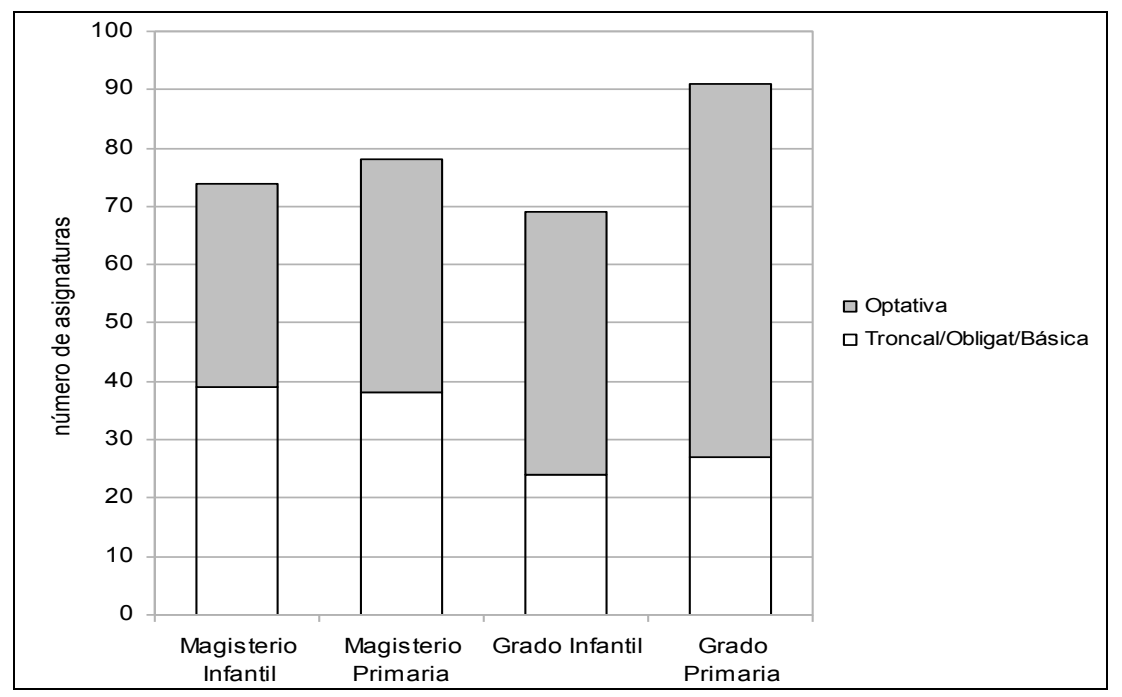

Figura 1. Número de asignaturas por titulación en el total nacional.

Otro aspecto observado es que, tal y como se observa en la Figura 1, el número de asignaturas "Básicas" relacionadas con las TIC en los nuevos grados es inferior a las asignaturas "Troncales" u "Obligatorias" de los antiguos Magisterios, mientras que, por el contrario, el peso de dichas asignaturas "Optativas" ha aumentado considerablemente en los nuevos Grados. Esto se debe, principalmente, al hecho de que ciertas universidades 
ofrecen menciones específicas que incluyen asignaturas relacionadas con las TIC. Asimismo, se observa una mayor oferta de asignaturas TIC en los Grados de Educación Primaria que en los de Educación Infantil, lo cual parece lógico ya que es en esta etapa donde se incluye como competencia básica el tratamiento de la información y competencia digital (Real Decreto 1513/2006, de 7 de diciembre).

De todo lo anterior se puede concluir que la formación en materias relacionadas con las TIC estará, principalmente, a criterio de los alumnos, no sólo por la elección de la universidad en la que estudiar, sino también dado el carácter de optatividad de la mayoría de las asignaturas TIC. Parece darse la situación señalada por Aguaded-Gómez, quién cuestiona el hecho de que en el ámbito intrauniversitario se decida si las TIC han de tener entidad como materias de formación para los futuros maestros o deban difuminarse como transversales en todo el ámbito curricular. En opinión de este autor, sólo si los futuros docentes aprenden los usos didácticos y posibilidades de explotación pedagógica podrán enseñarlas correctamente. Otros autores destacan el hecho de que, para que los docentes consideren las TIC como un recurso didáctico habitual en sus actividades de enseñanza y aprendizaje, es necesario formar a los docentes, no sólo con respecto al conocimiento y dominio de las herramientas informáticas, sino también a los beneficios didácticos que las mismas pueden reportarles (Quintero-Gallego y Hernández-Martín, 2005). A partir de estas reflexiones, se puede concluir que los egresados de aquellas universidades con poca o nula oferta de asignaturas específicas relacionadas con las TIC podrían carecer de la formación adecuada en dicha materia.

\section{CONCLUSIONES}

En España se han realizado grandes esfuerzos para adaptar la educación superior siguiendo el Proceso de Bolonia. Además de los cambios legislativos y procedimentales, tanto las universidades como la ANECA han venido trabajado durante los últimos años para crear el nuevo mapa de titulaciones adaptado al EEES. Este artículo describe el proceso de adaptación de las nuevas titulaciones de grado conducentes a ejercer la profesión de maestro, tanto en la especialidad de infantil como de primaria, profundizando en la presencia de asignaturas relacionadas con las TIC en los nuevos planes de estudio. Los resultados obtenidos muestran que, mientras los antiguos grados la asignatura troncal que ofrecía una formación común a todos los estudios dentro del territorio nacional, en los nuevos planes se observa una clara dispersión de la presencia de estas asignaturas dependiendo de cada universidad. Así, mientras algunas universidades incluyen varias asignaturas relacionadas con las TIC, o incluso menciones o itinerarios específicos en dicha materia, otras no ofertan asignatura alguna sobre TIC, ni siquiera como optatividad. Por otro lado, se observa como, en general, la presencia de asignaturas TIC en los Grados de Educación Primaria es mayor que en los de Educación Infantil. Este estudio, que puede extenderse a otros grados, puede ayudar a docentes, investigadores e incluso a los futuros estudiantes a conocer la oferta formativa que ofrecen los grados de magisterio de las universidades públicas españolas. 


\section{REFERENCIAS BIBLIOGRÁFICAS}

AGUADED-GÓMEZ, I. (2009) "Miopía en los nuevos planes de formación de maestros en España: ¿Docentes analógicos o digitales?”. En Comunicar, 33(XVII), 7-8.

ALONSO-MARTíN, P. (2008) "La formación en competencias del profesorado de magisterio en la especialidad de educación física", En Educar, 42, 63-77.

ANECA-LIBROS-BLANCOS (2010) "Libros blancos". [Recuperado el 18 de marzo de 2011 de: http://www.aneca.es/Documentos-y-publicaciones/Libros-blancos ]

ANECA-VERIFICA (2010) "Ordenación de las enseñanzas universitarias oficiales (grado y máster): Programa Verifica" [Recuperado el 18 de marzo de 2011 de: http://www.aneca.es/Programas/VERIFICA ]

CASTAÑEDA, L.J. (2009) "Las universidades apostando por las TIC: Modelos y paradojas de cambio institucional”. En EDUTEC, Revista Electrónica de Tecnología Educativa, 28, 1-14.

DECLARACIÓN DE BOLONIA (1999) [Recuperado el 18 de marzo de 2011 de: http://ec.europa.eu/education/policies/educ/bologna/bologna.pdf ]

eLEARNING (2004) "A programme for the effective integration of Information and Communication Technologies (ICT) in education and training systems in Europe", [Recuperado el 18 de marzo de 2011 de: http://ec.europa.eu/education/archive/elearning/programme_en.html ]

FERNÁNDEZ-MUÑOZ, R. (2003) "Competencias profesionales del docente en la sociedad del Siglo XXI". En Organización y Gestión Educativa: Revista del Forum Europeo de Administraciones de la Educación, 11(1), 4-7.

FERRO-SOTO, C., MARTÍNEZ-SENRA, A.I., OTERO-NEIRA, M.C. (2009) “Ventajas del uso de las TICs en el proceso de enseñanza-aprendizaje desde la óptica de los docentes universitarios españoles". En EDUTEC, Revista Electrónica de Tecnología Educativa, 29, 1-12.

LEY ORGÁNICA 2/2006, de 3 de mayo, de Educación (BOE no 106, de 4 de mayo de 2006).

MALDONADO-RICO, A.F. (2004) "Los títulos de grado de magisterio: El proceso de su diseño". En Revista Interuniversitaria de Formación del Profesorado, 18(3), 43-59.

MARCO DE CUALIFICACIONES (2005) "Un marco de cualificaciones para el Espacio Europeo de Educación Superior". [Recuperado el 18 de marzo de 2011, de: http://www.eees.es/pdf/QF-EHEA-May2005.pdf ]

ORDEN ECl/3854/2007 de 27 de diciembre por la que se establecen los requisitos para la verificación de los títulos universitarios oficiales que habiliten para el ejercicio de la profesión de Maestro en Educación Infantil (BOE no 312, de 29 de diciembre de 2007).

ORDEN ECl/3857/2007 de 27 de diciembre por la que se establecen los requisitos para la verificación de los títulos universitarios oficiales que habiliten para el ejercicio de la profesión de Maestro en Educación Primaria (BOE no 312, de 29 de diciembre de 2007).

PRENDES-ESPINOSA, M.P. (Dir) (2010) Competencias TIC para la docencia en la Universidad Pública Española: Indicadores y Propuestas para la Definición de Buenas Prácticas: 
Programa de Estudio y Análisis. Informe del Proyecto EA2009-0133 de la Secretaría de Estado de Universidades e Investigación. [Recuperado el 18 de marzo de 2011, de: http://www.um.es/competenciastic/informe.html ]

QUINTERO-GALLEGO, A., HERNÁNDEZ-MÁRTIN, A. (2005) "El profesor ante el reto de integrar las TIC en los procesos de enseñanza", En Enseñanza \& Teaching: Revista Interuniversitaria de Didáctica, 23, 305-321.

REAL DECRETO 1044/2003, de 1 de agosto, por el que se establece el procedimiento para la expedición por las universidades del Suplemento Europeo al Título (BOE no 218, de 11 de septiembre de 2003).

REAL DECRETO 1125/2003, de 5 de septiembre, por el que se establece el sistema europeo de créditos y el sistema de calificaciones en las titulaciones universitarias de carácter oficial y validez en todo el territorio nacional (BOE no224, de 18 de septiembre de 2003).

REAL DECRETO 1513/2006, de 7 de diciembre, por el que se establecen las enseñanzas mínimas de la Educación Primaria (BOE no 293, de 8 de diciembre de 2006).

REAL DECRETO 1630/2006, de 29 de diciembre, por el que se establecen las enseñanzas mínimas del segundo ciclo de Educación Infantil (BOE no 4, de 4 de enero de 2007).

REAL DECRETO 1393/2007, de 29 de octubre, por el que se establece la ordenación de las enseñanzas universitarias oficiales (BOE no 260, de 30 de octubre de 2007).

REAL DECRETO 1509/2008, de 12 de septiembre, por el que se regula el Registro de Universidades, Centros y Títulos (BOE no 260, de 30 de octubre de 2007).

RESOLUCIÓN de 17 de diciembre de 2007 por la que se publica el acuerdo del consejo de ministros por el que se establecen las condiciones a las que deben adecuarse los planes de estudios que habiliten para maestro en educación infantil y maestro en educación primaria (BOE no 305, de 21 de diciembre de 2007).

REYES-LÓPEZ, M.L. (2010) "Evaluación de los planes de estudio de formación del profesorado de educación musical: España". En Profesorado: Revista de Currículum y Formación del Profesorado, 14(2), 67-81.

SÁNCHEZ-LISSEN, E. (2002) "Elegir magisterio: Entre la motivación, la vocación y la obligación”. En Escuela Abierta: Revista de Investigación Educativa, 5, 125-146.

TUNING (2003) "Tuning Educational Structures in Europe", [Recuperado el 18 de marzo de 2011, de: http://tuning.unideusto.org/tuningeu/ ]

\section{Para citar este artículo:}

HERRADA, R. I.; HERRADA, G. (2011) «Adaptación de los estudios de magisterio al EEES:Las TIC en los nuevos planes de estudio" [artículo en línea]. EDUTEC, Revista Electrónica de Tecnología Educativa. Núm. 36 / Junio 2011. [Fecha de consulta: dd/mm/aa]. http://edutec.rediris.es/revelec2/revelec36

ISSN 1135-9250. 